\title{
Le choir du système Osiris, au centre du laboratoire de bactériologie
}

\author{
Entretien avec les Drs Françoise THERON et Jean-Claude MAS \\ LABM MAS, Marseille
}

\begin{abstract}
BioTribune : Pouvez-vous nous présenter brièvement votre laboratoire et en particulier votre activité en bactériologie?

Dr jean-Claude Mas : Notre laboratoire, qui a été créé dans une clinique, est un laboratoire à forte connotation hospitalière privée. La notion d'urgence et surtout de permanence des soins est très présente dans notre service ouvert $24 \mathrm{~h} / 24$. Une grande partie de la clientèle est celle de la clinique mais nous avons également une clientèle externe. La bactériologie est celle que l'on trouve habituellement dans un hôpital. Il s'agit d'une bactériologie à partir de malades traités, avec des germes à croissance souvent difficile. Par conséquent, nous avons beaucoup de prélèvements nobles (pièces opératoires, $L C R$, ponctions...). Nous travaillons en étroite collaboration avec les médecins avec lesquels nous examinons pratiquement tous les résultats particulièrement en ce qui concerne l'antibiogramme.
\end{abstract}

BioTribune : Quelle était l'organisation de votre laboratoire de bactériologie avant l'installation du système 0 siris?

Dr J.-C. Mas : Nous travaillons tout d'abord sur l'examen direct et la mise en culture. Les milieux utilisés sont choisis en fonction du Rémic, document de référence en bactériologie, en particulier pour les mises en culture. Nous suivons ainsi pas à pas ce qui est indiqué dans le Rémic. Pour ce qui est de l'identification, nous utilisons un système Vitek $l$, système automatique travaillant en milieu liquide et permettant de réaliser l'identification et l'antibiogramme du germe isolé. Le système Osiris est le complément du Vitek et permet de réal iser les antibiogrammes des bacilles Gram+, des Streptocoques et des Staphylocoques.

BioTribune : Que vous apporte 0 siris par rapport à votre automate de microdilution, Vitek 1 ? Quel est l'intérêt du module «Connectivity»?

Dr Françoise Théron : L'automate de microdilution (Vitek 1), intégré à notre organisation de bactériologie, a permis :

- une standardisation des identifications et antibiogrammes dans un contexte où nous avons des services de gardes et un personnel tournant,

- une certaine rapidité,

- une lecture automatique au temps de culture optimum.

II nous a paru utile de lui adjoindre un Osiris afin d'optimiser les antibiogrammes et l'étude des sensibilités bactériennes (notamment les Gram+, les germes exigeants et les anaérobies), qui nécessite un recours aux techniques de diffusion en gélose jusque là non automatisées. L'intérêt du module Connectivity est de permettre la centralisation sur Osiris des demandes et résultats de bactériologie et par là même, d'assurer un suivi épidémiologique des patients hospitalisés. Les connections relient Osiris au SIL, Osiris au Vitek en bidirectionnel. Dans le domaine de l'épidémiologie, nous sommes tenus dans le cadre de la lutte contre les infections nosocomiales de donner régulièrement un certain nombre de résultats. Ainsi, tous les trimestres ou chaque semaine, nous communiquons à des réseaux sentinelles, les taux de résistance à des antibiotiques pour les Staphylocoques dorés, les pyocyaniques et les entérobactéries isolées.

BioTribune : Après plusieurs mois d'utilisation, pouvez-vous nous dire quels ont été les bénéfices apportés par Osiris sur l'activité «antibiogrammes»?

DrF. Théron: Nous avons fiabilisé les résultats d'antibiogrammes en boîtes: lecture automatisée par la caméra ou le pied à coulisse, expertise des résultats en accord avec les dernières recommandations du CASFM permettant d'être à jour sur l'évolution des phénotypes de résistance.

La convivialité d' 0 siris permet de créer et de lancer des requêtes épidémiologiques «sur mesure» à la demande des cliniciens favo-

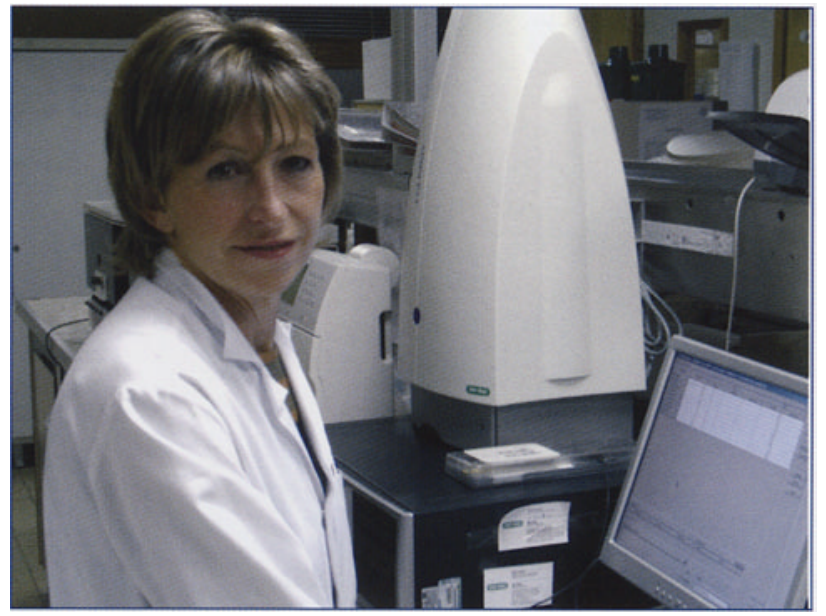

le Dr Françoise IHERON

risant ainsi le dialogue biologiste-praticiens. L'Osiris représente un système ouvert apportant la possibilité, à la différence de l'automate, de modifier instantanément la composition d'un antibiogramme si nécessaire. La sauvegarde et l'archivage des résultats sur CD facilement récupérable est aussi un plus.

\section{Le système OSIRIS}

Le système 0siris est un automate complet pour la lecture et l'interprétation des antibiogrammes en diffusion. II permet une lecture rapide et précise des résultats à l'aide d'une caméra ou mes en diffusion. II permet une lecture rapide et préécse des résult tats à 'raide d' une camera ou
d'un pied à coulisse et d'une interface logiciellie simple et conviviale couplée à une base de données fiable. Osiris étant très flexible, ii permet une personnalisation complète du système par rapport à l'organisation du laboratoire de bactériologie.

Les recommandations du comité français de l'antibiogramme (CA-SFM) sont réactualisées chaque année dans la base de données, afin de prendre en compte les nouvelles valeurs critiques des antibiotiques, les nouveiles interprétations des résultats, et d'apporter une aide pertinente aux antibiotiques, les nouvelies interpretations des résultats, et d' apporter une aide pertinente aux
cliniciens dans la mise en place d'un traitement thérapeutique approprié pour le patient. Afin d'optimiser ''utilisation du système Osiris au sein du laboratoire, une connexion à l'informatique centrale (SIL) peut-être mise en place, permettant ainsi la réception automatique des listes de travail et l'envoi des résultats.

D'autre part, Osiris permet une connexion bidirectionnelle directe avec les automates en milieu liquide afin d'intégrer les résultats expertisés de ces automates dans la base de données. Dans cette configuration, le système Osiris joue un rôle d'interface entre l'automate liquide et l'informatique du laboratoire (SLL) pour l'envoi des listes de travail et des résultats.

Cette configuration simplifie ainsi I' I'nterfaçage avec le SIL. L'ensemble des résultats des antibiogrammes en diffusion et liquides seront analysés avec le module Osiris Evolution, au travers de requêtes d'épidémiologie paramétrables. Le laboratoire pourra ainsi suivre l'écologie bactérienne, le taux de ésistance aux antibiotiques, l'évolution des bactéries multivi-résistantes (MRSA, BiLEE, le taux de résistance aux antibiotiques, l'évolution des bactéries multi-résistantes
Pseudo Ticar R...). Les rapports émis pourront également être transmis oux CLINs.

De plus, le module Osiris Evolution permet la mise en place d'un système d'alertes paramétrables pour le suivi des infections (infections nosocomiales...). Enfin, la mise en réseau du système Osiris avec l'installation de postes déportés offre une très grande souplesse d'utilisation (stations Osiris pour l l lecture des antibiogrammes, la validation des résultats ou 'analyse des données épidémiologiques).

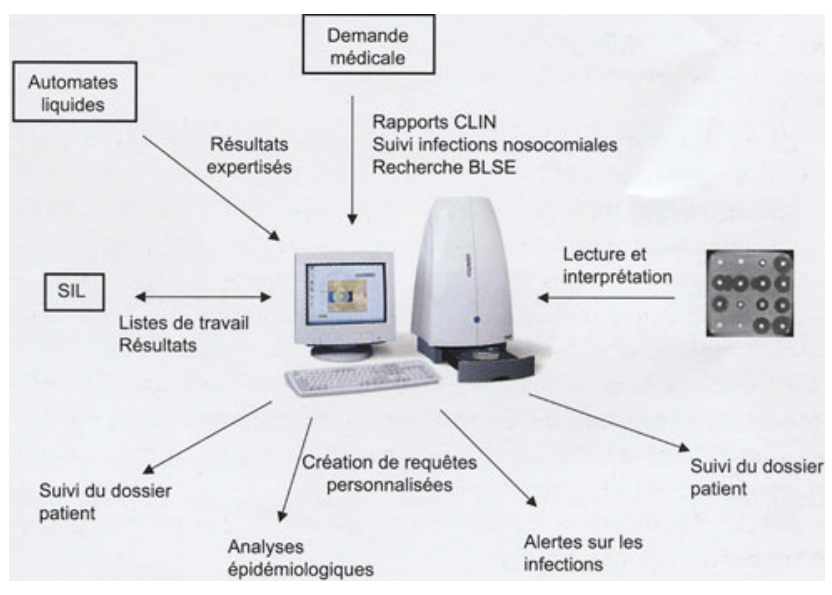

